\title{
Treatment of Large and Massive Rotator Cuff Tears: Does Infraspinatus Muscle Tear Affect Repair Integrity?
}

\author{
Sungwook Choi, Hyunchul Yang, Hyunseong Kang ${ }^{\square}$, Gyeong Min Kim
}

Department of Orthopedic Surgery, Jeju National University School of Medicine, Jeju, Korea

Background: Clinical outcomes and prognosis of large and massive rotator cuff tears are known to be unpredictable not only in degeneration of the rotator cuff, but also due to a high rate of retear.

Methods: Totally, 81 patients who had undergone arthroscopic rotator cuff repair from May 2008 to February 2016 were evaluated in our study. Clinical and functional evaluations were performed with the Constant score and the University of California, Los Angeles (UCLA) score, as well as full physical examination of the shoulder. All patients were confirmed to have magnetic resonance imaging (MRI) of tendon healing at least 1 year postoperatively.

Results: The average age at the time of surgery was 65 years (range, 47-78 years). The average duration of postoperative time in which a follow-up MRI was performed was 36.1 months (range, 12-110 months). Large tears were present in 48 cases (59.3\%) and massive tears in 33 cases (40.7\%). Overall, there were 33 retear cases (40.7\%). All the average clinical outcome scores were significantly improved at the last follow-up $(p<0.001)$, although repair integrity was not maintained. Compared to type A, types C, and D of the Collin's classification showed significantly higher retear rates $(p=0.036)$.

Conclusions: Arthroscopic rotator cuff repair yields improved clinical outcomes and a relatively high degree of patient satisfaction, despite the repair integrity not being maintained. Involvement of the subscapularis muscle or infraspinatus muscle had no effect on the retear rate.

(Clin Shoulder Elbow 2019;22(4):203-209)

Key Words: Rotator cuff; Arthroscopy; Shoulder

\section{Introduction}

Over the years, there have been numerous advances in surgical techniques of arthroscopic rotator cuff repair. However, the management of patients with large to massive rotator cuff tears remains a challenge for orthopedic surgeons. In the past, Goutallier et al. ${ }^{1}{ }^{1}$ devised a global fatty degeneration index (GFDI) and reported that a patient with grade 3 (as much muscle as fat) and grade 4 (less muscle than fat) fatty degeneration shows no improvement after rotator cuff repair. However, Burkhart et al. ${ }^{2)}$ reported that arthroscopic rotator cuff repair in patients with grade 3 or 4 fatty degeneration provides satisfactory functional improvement. As seen in previous reports, clinical outcomes and prognosis of large and massive rotator cuff tear are known to be unpredictable due to not only degeneration of rotator cuff, but also the high rate of retear.

Various factors influence the repair integrity, including patient age, tear size, and tendon quality. ${ }^{3)}$ In particular, higher preoperative fatty degeneration and larger tear sizes have a tendency to increase the retear rate. ${ }^{4)}$ It is critical to note that massive and irreparable rotator cuff tears should be considered as separate entities. ${ }^{5)}$ Massive tears are described as tears larger than $5 \mathrm{~cm}$, and tears involving two or more tendons. ${ }^{6,7}$

This study was undertaken to evaluate the clinical outcome

Received September 6, 2019. Revised November 2, 2019. Accepted November 3, 2019.

Correspondence to: Hyunseong Kang

Department of Orthopedic Surgery, Jeju National University Hospital, Jeju National University School of Medicine, 15 Aran 13-gil, Jeju 63241, Korea

Tel: +82-64-717-1690, Fax: +82-64-717-1131, E-mail: oskanghs@gmail.com, ORCID: https://orcid.org/0000-0001-6764-9929 IRB approval: Jeju National University Hospital (No. JNUH 2016-06-024).

Financial support: This work was supported by a research grant from Jeju National University Hospital in 2015. Conflict of interests: None. 
and maintenance of the repair integrity or retear rate of arthroscopic rotator cuff repair in patients with large and massive sized rotator cuff tears. Furthermore, we investigated the retear patterns in cases with structural failure after rotator cuff repair, with use of follow-up magnetic resonance imaging (MRI, Achieva 3.0T; Philips, Amsterdam, the Netherlands).

\section{Methods}

\section{Inclusion and Exclusion Criteria}

From May 2008 to February 2016, 203 patients with large and massive rotator cuff tears underwent arthroscopic rotator cuff repair at our institution. Inclusion criteria for the study were patients who had a large to massive rotator cuff tear confirmed by preoperative MRI, and who consequently underwent complete arthroscopic rotator cuff repair or partial repair. The study participants were further evaluated preoperatively and at least 1 year postoperatively for functional outcome. Patients with previous shoulder surgery were excluded. Patients were also excluded if they had labral tears, glenohumeral arthritis, or inflammatory disease. Complete subscapularis tendon tears were also excluded. Of the 203 patients enrolled, 84, 32, and 6 patients were excluded due to lack of follow-up MRI, a loss in followup, and use of patch augmentation during rotator cuff repair, respectively; the remaining 81 patients were included for the retrospective review.

\section{Patient Assessment}

The average patient age at the time of surgery was 65 years (range, 47-78 years), and postoperative follow-up duration was 36.1 months (range, 12-110 months). Patients enrolled included 28 male and 53 female, with the repairs being performed on 67 right and 14 left shoulders. Cuff tears in the dominant arm were seen in 73 cases, and in the nondominant arm in 8 cases. All patients had multiple tendon involvement.

History of trauma was reported by 17 patients; 49 patients were heavy workers, including farmers, carriers, and car mechanics, and 32 patients were required relatively low physical demands, such as housewives and office workers.

Arthroscopic suture bridge technique was applied in patients undergoing complete repair, whereas maximum partial repairs were performed in the remaining cases.

All patients were evaluated by the Constant score and the Shoulder Rating Scale of the University of California, Los Angeles (UCLA). Evaluations tear size, repair integrity, and fatty degeneration were performed preoperatively and at the last follow-up examination. ${ }^{8,9)}$

The initial size of rotator cuff tear was measured intraoperatively, based on the greatest dimension of tendon tear, and classified as follows: small, $<1 \mathrm{~cm}$; medium, 1 to $3 \mathrm{~cm}$; large, 3 to $5 \mathrm{~cm}$; or massive, $>5 \mathrm{~cm}$. Repair integrity of cuff muscles was evaluated with the classification devised by Sugaya et al., ${ }^{10)}$ through follow-up MRI: type I, sufficient thickness with homogeneous low intensity; type II, sufficient thickness with partial high intensity; type III, insufficient thickness without discontinuity; type IV, presence of a minor discontinuity; type $\mathrm{V}$, presence of a major discontinuity. Types IV and $\mathrm{V}$ were defined as retears. Fatty degeneration of the rotator cuff was assessed by preoperative MRI using the GFDI proposed by Goutallier et al."

The Collin's classification ${ }^{11)}$ was employed to structurally analyze the rotator cuff tear patterns. Collin et al. ${ }^{11)}$ classified rotator cuff tear patterns into five components: type $A$, supraspinatus and superior subscapularis tears; type $B$, supraspinatus and entire subscapularis tears; type $C$, supraspinatus, superior subscapularis, and infraspinatus tears; type $D$, supraspinatus and infraspinatus tears; and type E, supraspinatus, infraspinatus, and teres minor tears (Fig. 1). None of the cases presented with entire tear subscapularis muscle and teres minor muscle tear on the preoperative MRIs; hence, types B and E were excluded. This study therefore included patients classified as type A, C, and D (Table 1).

\section{Surgical Technique}

All surgical procedures were performed under general anesthesia, by a single surgeon. The position was not a complete lateral direction, but a semi-lateral position tilted 45 degrees, such that the patient's front face was diagonal. A diagnostic arthroscopic procedure was performed through posterior and anterior portals. The glenohumeral joint was evaluated for any arthritic changes and other accompanying intra-articular lesions. During surgery, the tear size, pattern, tendon quality, and presence of delamination were identified. Acromioplasty was completed for all patients. After evaluating mobility of the torn cuff, complete repair, wherever possible, was performed using the

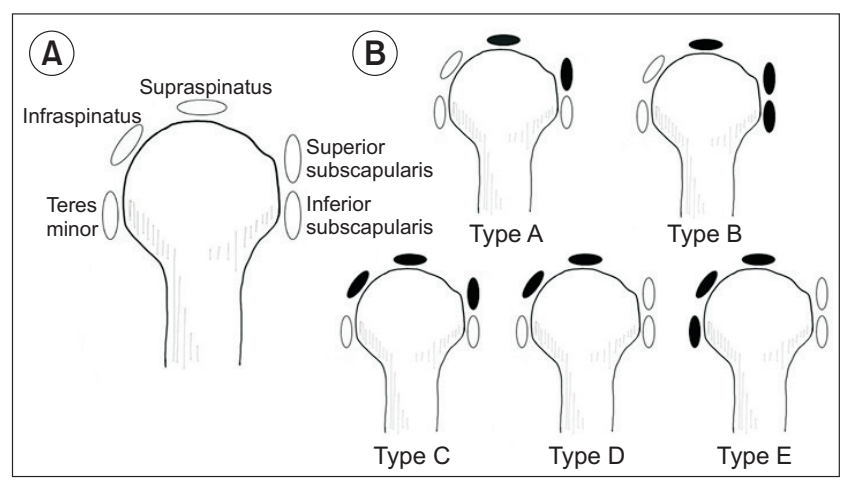

Fig. 1. (A) Five muscles that constitute the rotator cuff. (B) Collin's classification ${ }^{11)}$ classifies rotator cuff tear into five groups: type A, supraspinatus and superior subscapularis tears; type B, supraspinatus and entire subscapularis tears; type $C$, supraspinatus, superior subscapularis, and infraspinatus tears; type $\mathrm{D}$, supraspinatus and infraspinatus tears; and type E, supraspinatus, infraspinatus, and teres minor tears. 
Table 1. Patient and Tear Demographics

\begin{tabular}{|c|c|c|c|c|c|c|c|}
\hline \multirow{2}{*}{$\begin{array}{l}\text { Intraoperative } \\
\text { tear size }\end{array}$} & \multicolumn{2}{|c|}{ Sex } & \multicolumn{2}{|c|}{ Age (yr) } & \multicolumn{3}{|c|}{ Collin's classification } \\
\hline & Male & Female & $<65$ & $\geq 65$ & Type A & Type C & Type D \\
\hline Large $(\mathrm{n}=48)$ & $13(27.1)$ & $35(72.9)$ & $18(37.5)$ & $30(62.5)$ & $8(16.7)$ & $9(18.8)$ & $31(64.6)$ \\
\hline Massive $(\mathrm{n}=33)$ & $15(45.5)$ & $18(54.5)$ & $17(51.5)$ & $16(48.5)$ & $7(21.2)$ & $17(51.5)$ & $9(27.3)$ \\
\hline Overall (n=81) & $28(34.6)$ & $53(65.4)$ & $35(43.2)$ & $46(56.8)$ & $15(18.5)$ & $26(32.1)$ & $40(49.4)$ \\
\hline
\end{tabular}

Values are presented as number (\%).
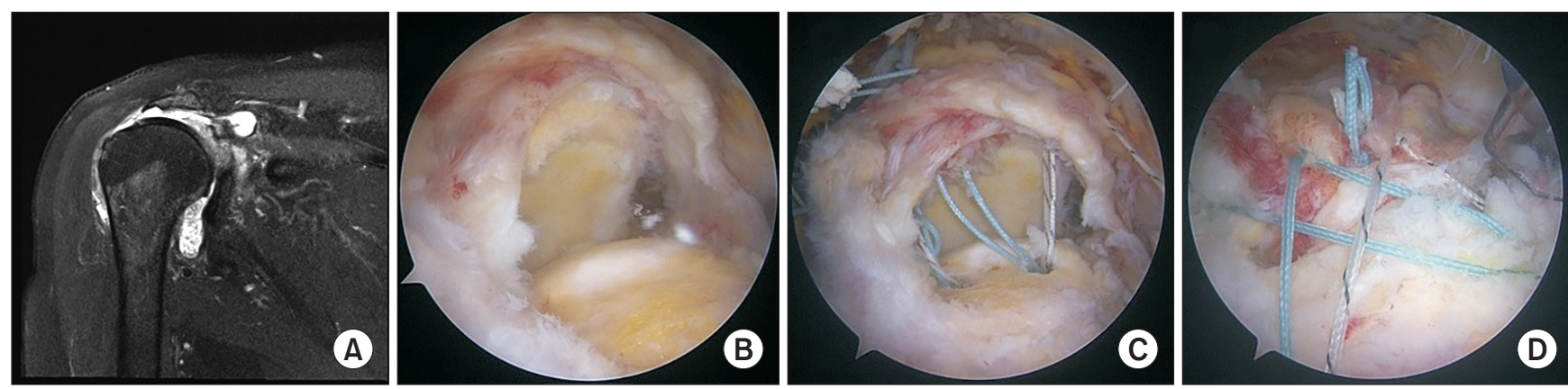

Fig. 2. (A) Preoperative coronal T2-weighted image showing a full-thickness rotator cuff tear with substantial muscle retraction. (B) The arthroscopic view showing massive rotator cuff tear. Degeneration of rotator cuff is severe and glenoid is exposed. (C) Suture anchors were placed to repair the rotator cuff on the footprint. (D) A repair configuration after arthroscopic rotator cuff repair. Due to the extensive rupture of the rotator cuff, the footprint was not completely covered.

double-row suture technique.

In the double-row suture technique, medial row anchors (3.7 $\mathrm{mm}$ and $4.5 \mathrm{~mm}$ Bio-Corkscrew suture anchor; Arthrex, Naples, $\mathrm{FL}, \mathrm{USA}$ ) were first placed in a location just lateral to the articular surface of the humeral head, considered as the medial edge of the footprint. Next, either a Scorpion (Arthrex) or suture hook (Linvatec, Largo, FL, USA) was used to pass the suture through the supraspinatus tendon, near the musculotendinous junction. The knots were tied on the medial row, and the tendon was reduced into the bone. Lateral row anchors $(3.5 \mathrm{~mm}$ and 4.5 $\mathrm{mm}$ Bio-PushLock anchor or SwiveLock; Arthrex) were placed 5 to $10 \mathrm{~mm}$ lateral to the greater tuberosity after preparing the bone sockets. The number of anchors and selection of anchor size depended on the tear size, bone quality of the footprint, and anchor stability. The delamination tendon was repaired after passing the thread through the suture hook as much as possible.

In case of incomplete coverage of the foot print, margin convergence sutures were applied first and the torn tendon was pulled to cover the foot print to the maximum possible; suture anchors were subsequently placed to the bone by the single-row fixation technique. Biceps tenotomy or tenodesis was performed in patients with severe degeneration of the biceps tendon. Biceps tenotomy was performed for patients older than age 65 years, whereas tenodesis was performed for patients younger than age 65 years. Patients with biceps partial tear underwent only debridement (Fig. 2).

\section{Postoperative Rehabilitation}

Patients were immobilized during the 6 weeks of rehabilitation by applying an abduction brace. During this time, the patient was permitted to keep the arm out of the brace only during exercises. All patients started pendulum exercise on the day after surgery. Patients with large tears started passive forward flexion on the third postoperative day using the continuous passive movement machine (ORMED GmbH, Freiburg, Germany). Patients with massive tears began rehabilitation 1 week postoperatively. Active exercise was not allowed until 6 weeks postoperatively, after which active exercise of the shoulder was slightly increased.

\section{Statistical Analysis}

The paired t-test was performed to evaluate functional scores between the preoperative and postoperative results. Pearson $\chi^{2}$ test was performed to assess the correlation between repair integrity and other factors. Pearson $\chi^{2}$ test and ANOVA test were applied to analyze structural factors of the rotator cuff. Statistical significance was set at $p$-value less than 0.05. All statistics were analyzed with the PASW software package (ver. 18.0; IBM Corp., Armonk, NY, USA).

\section{Results}

All functional scores showed significant improvement over the preoperative scores at the most recent follow-up. The mean 
UCLA score increased from a preoperative mean of $15.17 \pm$ 4.09 points to $30.26 \pm 3.50$ points $(p<0.001)$, and the Constant score improved from a preoperative mean of $54.68 \pm 12.48$ points to $82.49 \pm 10.32$ points $(p<0.001)$ at the last follow-up (Table 2).

Repair integrity evaluation by the Sugaya classification with MRI revealed type I healing in 6 cases (7.4\%), type II in 30 cases (37.0\%), type III in 12 cases (14.8\%), type IV in 8 cases (9.9\%), and type $V$ in 25 cases (30.9\%). The overall number of retears (types IV and V) was 33 cases (40.7\%). The retear rate was $22.9 \%$ (11 of 48 ) in large tears, and $66.7 \%$ (22 of 33 ) in massive tears. A larger intraoperative tear size was associated with a statistically significant higher rate of retear ( $p<0.001$; Fig. 3).

The average follow-up period of patients with maintained repair integrity was 36.2 months, and for patients with retear was 36.1 months, which were statistically not different ( $p<0.988$ ).

Preoperative GFDI was confirmed by MRI: 2 cases were determined to be grade 1 and had 1 retear; 29 cases showed grade 2 with 3 retears (10.3\%). A retear was found in 14 of 32 grade 3 cases $(43.8 \%)$, and 15 retears were observed in 18 grade 4 cases $(83.3 \%)$. Higher preoperative fatty degeneration grades were associated with increased incidence of retear $(p<0.001$; Fig. 4).
Collin's classification was also confirmed by MRI; 15 cases were determined as type A with 2 retears (13.3\%), 26 type C cases had 17 retears (65.4\%), and 40 type D cases had 14 retears (35.0\%). To evaluate whether infraspinatus tear affected repair integrity, we compared two groups, type $A$ with type $C$ and D. Although there were few type A cases, a statistically significant difference was obtained in the repair integrity between the two groups ( $p=0.036$; Fig. 5). For each comparative analysis using ANOVA test, type $C$ had more retears than A $(p<0.001)$ and D $(p<0.03)$. However, there was no statistically significant difference between types $A$ and $D$, although $D$ tended to retear more than A ( $p=0.28$; Table 3).

\section{Discussion}

The goal of arthroscopic rotator cuff repair is to maintain the mechanical strength to avoid retears due to repeated stress in daily life. Therefore, it is important to maintain the integrity of the repaired rotator cuff until complete healing is achieved. Arthroscopic rotator cuff repair results in significant improvement of shoulder pain and function. ${ }^{4,12,13)}$ It is equally critical to plan repair strategies before surgery, and MRI is the most useful tool for assessing severity of the tear preoperatively. Yoo et al. ${ }^{14)}$

Table 2. Clinical and Radiologic Outcomes

\begin{tabular}{|c|c|c|c|c|c|c|c|c|}
\hline \multirow{2}{*}{$\begin{array}{c}\text { Intraoperative } \\
\text { tear size }\end{array}$} & \multicolumn{3}{|c|}{ UCLA score $^{*}$} & \multicolumn{3}{|c|}{ Constant score } & \multicolumn{2}{|c|}{ Retear } \\
\hline & Preop. & Postop. & $p$-value & Preop. & Postop. & $p$-value & Intact & Retear \\
\hline Large $(n=48)$ & $15.71 \pm 3.96$ & $30.63 \pm 3.48$ & $<0.001$ & $55.58 \pm 13.42$ & $83.21 \pm 11.72$ & $<0.001$ & $37(77.1)$ & $11(22.9)$ \\
\hline Massive $(\mathrm{n}=33)$ & $14.39 \pm 4.22$ & $29.73 \pm 3.52$ & $<0.001$ & $53.36 \pm 11.05$ & $81.45 \pm 7.92$ & $<0.001$ & $11(33.3)$ & $22(66.7)$ \\
\hline Overall $(\mathrm{n}=81)$ & $15.17 \pm 4.09$ & $30.26 \pm 3.50$ & $<0.001$ & $54.68 \pm 12.48$ & $82.49 \pm 10.32$ & $<0.001$ & $48(59.3)$ & $33(40.7)$ \\
\hline
\end{tabular}

Values are presented as mean \pm standard deviation or number (\%).

Preop.: preoperative, Postop.: postoperative.

*The Shoulder Rating Scale of the University of California, Los Angeles (UCLA) score.

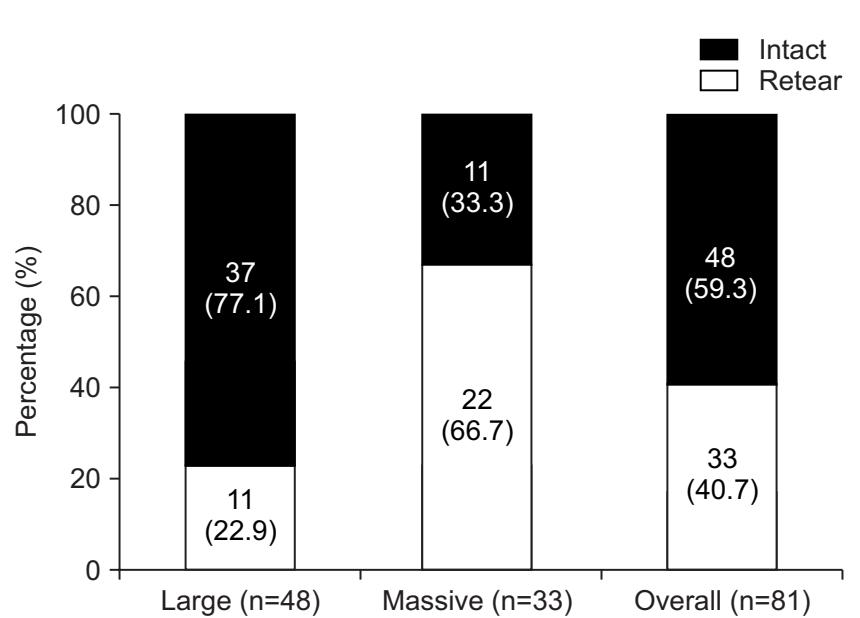

Fig. 3. Correlation between preoperative tear size and repair integrity $(p<0.001)$.

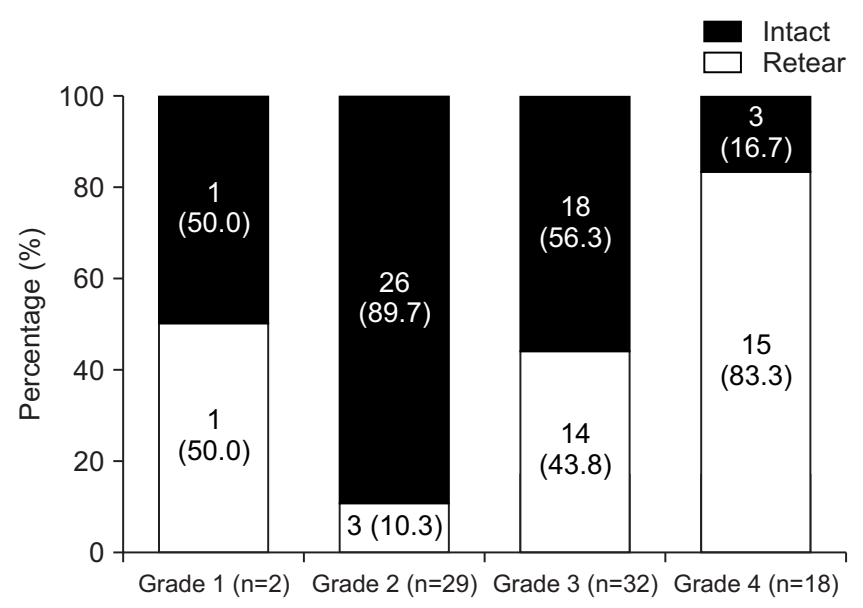

Fig. 4. Correlation between global fatty degeneration index (GFDI) and repair integrity $(p<0.001)$. 


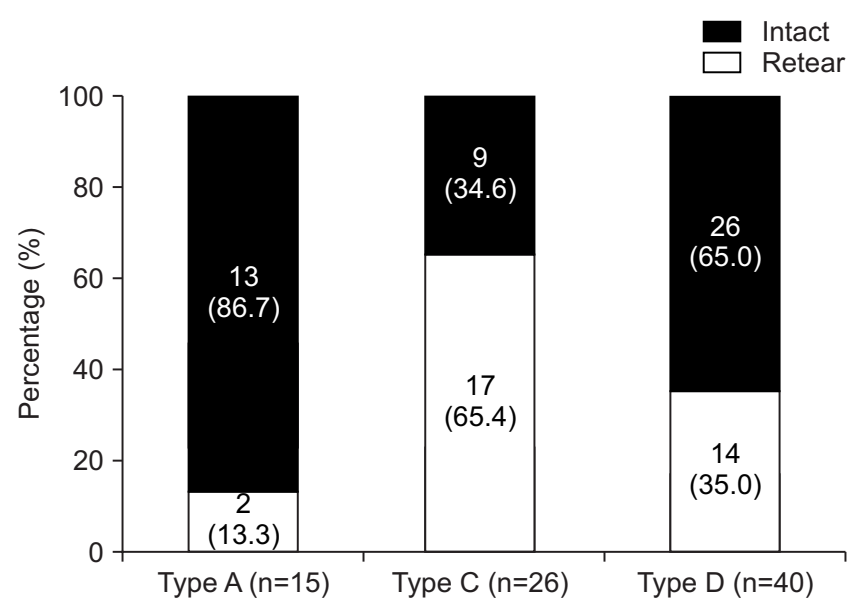

Fig. 5. Correlation between Collin's classification and repair integrity $(p=0.036)$.

identified that sagittal tear size of $>32 \mathrm{~mm}$ and coronal tear size of $>31 \mathrm{~mm}$ on MRI are associated with the inability to obtain an anatomical repair. As mentioned previously, severe fatty degeneration correlates with unsatisfactory surgical outcomes and higher retear rate. ${ }^{1,15)}$ However, with advances in surgical techniques, numerous researches report a significant improvement in the clinical outcomes of patients with large to massive rotator cuff tears. ${ }^{2,16-18)}$ Since most large to massive rotator cuff tears encompass the supraspinatus muscle, the fatty degeneration of supraspinatus muscle is associated with the surgical outcome.

Park et al. ${ }^{12)}$ reported that repair of large to massive rotator cuff tears using the double-row technique is superior to the single-row method. We previously reported that retear rates are higher in cases of larger intraoperative tear size and higher preoperative grade of fatty degeneration. ${ }^{4)}$ We obtained similar results in the current study for patients with large to massive rotator cuff tears.

Although the repair integrity was not maintained, patients were satisfied with surgical outcomes and displayed improved functional scores. These results are similar to the report of Burkhart et al., ${ }^{2)}$ but the previous study lacked analysis of the postoperative tendon status. Paxton et al. ${ }^{19)}$ reported that clinical improvement and pain relief after arthroscopic rotator cuff repair of large and massive tears are durable at the time of longterm follow-up (10 years), despite early retears. These results remained unchanged in spite of radiographic progression of arthropathy.

Furthermore, Cho et al. ${ }^{20,21)}$ reported that medial cuff failure is a frequent retear pattern and mentioned various causes of retears, including poor-quality tendon tissue and suture breakage. Similarly, retears at the musculocutaneous junction were commonly observed in our study.

Recently, Miller et al. ${ }^{22)}$ proposed that better characterization of timing the structural failure of rotator cuff repairs would help identify weaknesses in repair strategies. He reported that early
Table 3. Multiple Comparisons by Collin's Classification

\begin{tabular}{lcccc}
\hline $\begin{array}{c}\text { Comparison of } \\
\text { involved tendon }\end{array}$ & Mean difference & Lower bound & Upper bound & $p$-value* \\
\hline Type C-A & 0.52 & 0.16 & 0.88 & 0.00 \\
Type D-A & 0.22 & -0.12 & 0.55 & 0.28 \\
\hline Type D-C & -0.30 & -0.52 & -0.03 & 0.03 \\
\hline
\end{tabular}

${ }^{*}$ Adjusted $p$-value.

retears were due to mechanical failure, intraoperative fixation deficiencies, poor compliance with postoperative immobilization, and excessive tension at the repair site; conversely, late retears were due to biologic failure, alterations in the initial biologic healing environment, and medical comorbidities. In our study, it was difficult to identify actual timing of the retear of rotator cuff retear. Further researches to identify timing of retears would help plan better repair strategies.

Park et al. ${ }^{23)}$ reported that grade II and higher infraspinatus fatty degeneration correlated with a higher failure rate in small and medium tear patients. We used the Collin's classification to classify the patient group to determine whether torn infraspinatus muscles affect the outcome of surgery. It is more likely to involve multiple tendons rather than just one tendon in a large to massive rotator cuff tear. Therefore, the authors figured that comparison among a group of affected tendons was clinically more significant than identification of outcomes of a single tendon tear.

In our study, types C and D involving infraspinatus muscles had a higher retear rate than type $A$ (comprising supraspinatus muscle and subscapularis muscle). However, when type A, C, and $D$ were compared using ANOVA, type $C$ had a higher retear rate than $A$ and $D$, but there was no significant difference between A and D. All three muscles are involved in type C, which might affect the high retear rate. Although type $D$ had a higher retear rate than type $A$, there was no statistically significant difference.

There were several limitations to this study. First, the experimental design was a retrospective review with no control group, and was not a randomized controlled blind trial. Second, postoperative repair integrity evaluation by MRI was not conducted in all cases, which may have resulted in a subtle selection bias. Third, the surgery was not performed only with the double-row suture technique, but also with single row suture technique, marginal convergence, biceps tenodesis or tenotomy, depending on the tear size and quality. It is possible that these differences in surgical methods may have influenced the outcomes. Fourth, the follow-up periods in some patients were relatively short. Lastly, multivariate logistic regression analysis needs to be considered for identifying other factors in addition to the involved tendon; however, this study has a small number of patients. 


\section{Conclusion}

The retear rate was $40.7 \%$ for patients with large and massive tear size, but the clinical symptoms of the patients with or without retear showed good results. Arthroscopic rotator cuff repair yields improved clinical outcomes and a relatively high degree of patient satisfaction, despite difficulty in maintaining the repair integrity. The degree of fatty degeneration and higher number of involved tendons influences the high retear rate. There was no difference in the effect of subscapularis muscle or infraspinatus muscle involvement on the retear rate.

\section{References}

1. Goutallier D, Postel JM, Gleyze P, Leguilloux P, Van Driessche S. Influence of cuff muscle fatty degeneration on anatomic and functional outcomes after simple suture of full-thickness tears. J Shoulder Elbow Surg. 2003;12(6):550-4. doi: 10.1016/s10582746(03)00211-8.

2. Burkhart SS, Barth JR, Richards DP, Zlatkin MB, Larsen M. Arthroscopic repair of massive rotator cuff tears with stage 3 and 4 fatty degeneration. Arthroscopy. 2007;23(4):347-54. doi: 10.1016/j.arthro.2006.12.012.

3. Boileau P, Brassart N, Watkinson DJ, Carles M, Hatzidakis AM, Krishnan SG. Arthroscopic repair of full-thickness tears of the supraspinatus: does the tendon really heal? J Bone Joint Surg Am. 2005;87(6):1229-40. doi: 10.2106/JBJS.D.02035.

4. Choi S, Kim MK, Kim GM, Roh YH, Hwang IK, Kang H. Factors associated with clinical and structural outcomes after arthroscopic rotator cuff repair with a suture bridge technique in medium, large, and massive tears. J Shoulder Elbow Surg. 2014;23(11):1675-81. doi: 10.1016/j.jse.2014.02.021.

5. Anley CM, Chan SK, Snow M. Arthroscopic treatment options for irreparable rotator cuff tears of the shoulder. World J Orthop. 2014;5(5):557-65. doi: 10.5312/wjo.v5.i5.557.

6. Cofield RH, Parvizi J, Hoffmeyer PJ, Lanzer WL, Ilstrup DM, Rowland CM. Surgical repair of chronic rotator cuff tears. A prospective long-term study. J Bone Joint Surg Am. 2001; 83(1):71-7. doi: 10.2106/00004623-200101000-00010.

7. Gerber C, Fuchs B, Hodler J. The results of repair of massive tears of the rotator cuff. J Bone Joint Surg Am. 2000;82(4):50515. doi: 10.2106/00004623-200004000-00006.

8. Constant CR, Murley AH. A clinical method of functional assessment of the shoulder. Clin Orthop Relat Res. 1987; (214):160-4.

9. Duquin TR, Buyea C, Bisson LJ. Which method of rotator cuff repair leads to the highest rate of structural healing? A systematic review. Am J Sports Med. 2010;38(4):835-41. doi: 10.1177/0363546509359679.

10. Sugaya H, Maeda K, Matsuki K, Moriishi J. Repair integrity and functional outcome after arthroscopic double-row rotator cuff repair. A prospective outcome study. J Bone Joint Surg Am. 2007;89(5):953-60. doi: 10.2106/JBJS.F.00512.

11. Collin P, Matsumura N, Lädermann A, Denard PJ, Walch G. Relationship between massive chronic rotator cuff tear pattern and loss of active shoulder range of motion. J Shoulder Elbow Surg. 2014;23(8):1195-202. doi: 10.1016/j.jse.2013.11.019.

12. Park JY, Lhee SH, Choi JH, Park HK, Yu JW, Seo JB. Comparison of the clinical outcomes of single- and double-row repairs in rotator cuff tears. Am J Sports Med. 2008;36(7):1310-6. doi: 10.1177/0363546508315039.

13. Neri BR, Chan KW, Kwon YW. Management of massive and irreparable rotator cuff tears. J Shoulder Elbow Surg. 2009;18(5):808-18. doi: 10.1016/j.jse.2009.03.013.

14. Yoo JC, Ahn JH, Yang JH, Koh KH, Choi SH, Yoon YC. Correlation of arthroscopic repairability of large to massive rotator cuff tears with preoperative magnetic resonance imaging scans. Arthroscopy. 2009;25(6):573-82. doi: 10.1016/ j.arthro.2008.12.015.

15. Bedi A, Dines J, Warren RF, Dines DM. Massive tears of the rotator cuff. J Bone Joint Surg Am. 2010;92(9):1894-908. doi: 10.2106/JBJS.I.01531.

16. Berhouet J, Collin P, Benkalfate T, et al.; Société d'Orthopédie de l'Ouest. Massive rotator cuff tears in patients younger than 65 years. Epidemiology and characteristics. Orthop Traumatol Surg Res. 2009;95(4 Suppl 1):S13-8. doi: 10.1016/ j.otsr.2009.03.006.

17. Carbonel I, Martínez AA, Aldea E, Ripalda J, Herrera A. Outcome and structural integrity of rotator cuff after arthroscopic treatment of large and massive tears with double row technique: a 2-year followup. Adv Orthop. 2013;2013:914148. doi: 10.1155/2013/914148.

18. Favard L, Berhouet J, Colmar M, et al.; Société d'orthopédie de l'Ouest. Massive rotator cuff tears in patients younger than 65 years. What treatment options are available? Orthop Traumatol Surg Res. 2009;95(4 Suppl 1):S19-26. doi: 10.1016/ j.otsr.2009.03.005.

19. Paxton ES, Teefey SA, Dahiya N, Keener JD, Yamaguchi K, Galatz LM. Clinical and radiographic outcomes of failed repairs of large or massive rotator cuff tears: minimum ten-year follow-up. J Bone Joint Surg Am. 2013;95(7):627-32. doi: 10.2106/JBJS.L.00255.

20. Cho NS, Lee BG, Rhee YG. Arthroscopic rotator cuff repair using a suture bridge technique: is the repair integrity actually maintained? Am J Sports Med. 2011;39(10):2108-16. doi: 10.1177/0363546510397171.

21. Cho NS, Yi JW, Lee BG, Rhee YG. Retear patterns after arthroscopic rotator cuff repair: single-row versus suture bridge technique. Am J Sports Med. 2010;38(4):664-71. doi: 10.1177/0363546509350081.

22. Miller BS, Downie BK, Kohen RB, et al. When do rotator cuff repairs fail? Serial ultrasound examination after arthroscopic 
repair of large and massive rotator cuff tears. Am J Sports Med. 2011;39(10):2064-70. doi: 10.1177/0363546511413372.

23. Park JS, Park HJ, Kim SH, Oh JH. Prognostic factors affecting rotator cuff healing after arthroscopic repair in small to medium-sized tears. Am J Sports Med. 2015;43(10):2386-92. doi: 10.1177/0363546515594449. 